\title{
Eau et aménagement du territoire : rapport général
}

\author{
Water and Regional Development and Planning
}

\author{
par A. Bergeret \\ Directeur Adjoint d'EDF - CNEH
}

This general report analyses the 20 papers proposed for the question " Water and regional development and planning ". Firstly, the author locates the papers in the context of the congress and examines the participation of the French water school to the world development in the area of regional development and planning. It highlights the universality of the research concerning this sector.

After a classification of the papers, it analyses with details each one.

\section{PROLOGUE}

Qu'est-ce que l'Ecole Française de l'Eau apporte au développement du monde dans le domaine de l'aménagement du territoire ? Comment les études et les travaux des chercheurs, des ingénieurs, des maîtres d'ouvrage et des services de l'Etat peuvent-ils être transposés dans d'autres pays ? Comment notre savoir et notre expérience dans la conception et la gestion des ouvrages d'aménagement des rivières peuvent-ils être utilisés avec profit pour l'humanité dans d'autres territoires, sous d'autres climats, dans d'autres législations que les nôtres?

La réponse à ces interrogations n'est pas immédiate et dans les communications qui sont au cœur de ce rapport, il faut souvent lire entre les lignes pour découvrir ce que cette activité de recherche a d'universel et ce qu'elle peut apporter à la communauté mondiale.

Pour éclairer le paysage et planter le décor, il est utile tout d'abord de situer la communauté scientifique et industrielle française dans le monde d'aujourd'hui et d'examiner comment elle s'y exprime, puis de mettre en évidence le caractère universel du milieu naturel sur lequel elle exerce son activité et enfin de vérifier que les orientations de ces travaux de recherche correspondent aux préoccupations communes à tous les pays du monde, pour un développement durable de l'humanité.

\subsection{Le monde actuel : " pour une petite planète" :}

Les progrès scientifiques du XXème siècle qui s'achève ont ouvert une ère nouvelle où le temps et l'espace ont été pratiquement abolis. Nous pouvons nous transporter d'un point à l'autre du globe terrestre presque aussi rapidement que pour aller au travail chaque matin. Nous communiquons instantanément des données et des informations à l'autre bout du monde.

Dans une économie devenue mondiale, le marché du travail s'est élargi à l'échelle de la terre et les ingénieurs de l'hydraulique sillonnent le monde au gré des contrats pour prêter leur concours aux pays en voie de développement et $y$ diffuser leurs idées et leur pratique. Les chercheurs de tous les pays dialoguent sur le réseau des réseaux Internet et vont chercher dans la Toile les informations contenues dans cette gigantesque bibliothèque planétaire.

Les symposiums et les colloques se multiplient aux quatre coins du monde et dans ces lieux d'échange les universitaires, les chercheurs, les ingénieurs, les responsables de l'Etat et des secteurs publics et privés, exposent les résultats de leur recherche, font valoir la pertinence de leurs algorithmes et la qualité de leurs logiciels de modélisation, font découvrir leurs inventions ou de nouvelles méthodes, propagent leurs idées et apportent ainsi leur concours à l'aménagement de la planète Terre.

1.2. L'aménagement de la planète Terre : le temps et l'espace maîtrisés

Cette recherche et ces études reposent sur la connaissance du milieu naturel et sur la manière d'utiliser efficacement ses ressources. Elles s'appuient sur la collecte et l'analyse de données multiples : météorologiques, topographiques, hydrologiques, géologiques et géophysiques. Elles visent à maîtriser les fleuves et les rivières. Elles recherchent des méthodes de gestion de l'eau satisfaisant à la fois les besoins d'irrigation, de production d'énergie, d'alimentation d'eau potable, de loisirs et la nécessité de préserver l'environnement. 
Et les experts de ces disciplines sont d'emblée familiers avec leurs homologues de tous les pays du monde, parce qu'ils parlent une langue universelle et éternelle, celle de la pluie et des nuages, celle des ruisseaux et des fleuves, celles des plaines et des montagnes, celle de la terre et de l'eau, celle de l'espace et du temps maîtrisés pour un développement durable de l'humanité.

\subsection{Le développement durable : pour un monde meilleur}

Le développement implique la maîtrise des ressources en eau, le règlement des problèmes liés au partage de cette ressource et par la maîtrise des risques de pénurie ou d'inondation.

La récente Conférence Internationale de Paris sur le thème : " Eau et développement durable ", a mis au centre des débats cette préoccupation vitale pour l'humanité.

Dans les pays dits développés, la prise de conscience de la rareté de l'eau, de l'inégalité spatiale et temporelle de cette ressource et de la nécessité de préserver l'environnement et de maîtriser les risques, se traduit par un investissement intellectuel important dans la recherche de solutions et par l'engagement de dépenses pour les mettre en application. Ces études et ces travaux sont essentiellement financés par les budgets publics de ces pays sous la pression de la Société qui, devant les progrès prodigieux de la science et le développement des outils informatiques, accepte de moins en moins que l'Etat et les Collectivités ne puissent prévoir et limiter les catastrophes provoquées par l'eau, ainsi que les atteintes à l'environnement.

Dans les pays en voie de développement, l'enjeu est le même mais d'un autre ordre car les populations de ces pays ont besoin d'eau potable, de quoi irriguer leurs cultures ou mettre en valeur leur potentiel hydroélectrique. Elles ont le droit de vivre mieux et surtout de mourir en moins grand nombre de faim, de soif ou par suite de catastrophes provoquées par l'eau.

Collecter les données météorologiques et hydrologiques, prévoir les crues, délimiter les zones d'inondation, créer des réservoirs pour irriguer les terres ou fournir de l'énergie, ou écrêter les crues, tout cela fait appel aux mêmes concepts et aux mêmes outils de modélisation et de gestion qu'il s'agisse de la Loire ou de la Vistule, qu'on se trouve en AdourGaronne ou dans la région de Singrali en Inde, à Vaison la Romaine ou à Shangaii, à la Réunion ou au Bangladesh, en Bolivie ou en Maurienne, au Burkina-Faso ou en France.

$\mathrm{Vu}$ de façon un peu globale, voilà ce que l'on peut lire en diagonale dans les textes qui ont été analysés et qui démontrent que l'Ecole Française de l'Eau ne démérite pas de son passé et continue à apporter sa contribution au développement mondial.

Ce tableau général étant brossé et les communications étant maintenant situées dans le thème des 25èmes Journées de l'Hydraulique, il est temps d'essayer de dégager une synthèse des travaux de recherche ou d'études présentés avant de procéder au rapport détaillé des communications.

\section{II — SYNTHÈSE}

Près de cinquante communications ont été proposées sur la question de l'eau et de l'aménagement du territoire mais seulement la moitié d'entre elles a pu être retenue comme support de ce rapport général.
Les vingt rapports analysés ont été classés en cinq thèmes touchant à la connaissance de la ressource en eau, à la gestion des crues et des étiages, à la gestion en dynamique des cours d'eau, à la gestion des écosystèmes aquatiques et enfin à la gestion opérationnelle de l'eau. Certaines communications contiennent des sujets pouvant être rattachés à plusieurs thèmes.

Cet afflux record de sujets est significatif de l'arrivée à maturité de projets de recherche lancés depuis plusieurs années par des donneurs d'ordre nationaux et internationaux et rendus possibles grâce à l'explosion des moyens informatiques de calcul, des possibilités de modélisation numérique et des moyens de collecte et de transmission des données et des informations.

Une grande partie des contributions s'inscrit dans des programmes d'études lancés en France à la suite de la Loi sur l'Eau de 1992 par des aménageurs, des gestionnaires de l'eau, des services publics comme les Agences de l'eau, les DIREN, EDF, la CNR, I'EPALA, le Cemagref.

Ces responsables de l'aménagement du territoire ont besoin à la fois d'outils de gestion stratégique pour planifier et définir les futurs aménagements ou pour corriger les aménagements existants, et également d'outils pour gérer en temps réel ces ouvrages ou pour alerter et mettre en sécurité les riverains et utilisateurs des cours d'eau.

Ces outils entrent pour la plupart dans une phase opérationnelle sur le territoire français mais les applications à l'international sont plutôt rares et c'est bien dommage car l'ouverture vers l'international est un facteur de motivation et d'enrichissement comme le démontrent certaines communications émanant de programmes de recherche européens comme EUREKA ou d'organisations non gouvernementales ou encore de développements en partenariat avec des universités et des sociétés étrangères.

L'Ecole Française de l'Eau ne manque certainement pas de compétences ni de faculté d'adaptation pour s'intégrer dans les actions de recherche et les études à l'international.

C'est ce que nous allons maintenant démontrer en analysant dans le détail chaque contribution.

\section{III — RAPPORT DÉTAILLÉ}

\section{- 3.2 Connaissance quantitative de la ressource}

Sur le thème de l'évaluation de la ressource en eau, nous avons retenu quatre rapports, tous excellents et originaux.

Trois d'entre eux caractérisent les avancées de la recherche dans ce domaine très difficile où les gestionnaires et aménageurs du territoire attendent de la science qu'elle puisse prédéterminer avec précision les débits générés par les précipitations pluviales en tout point d'une zone géographique donnée.

Ces trois communications de MM. J. LAVABRE, P. ARNAUD et N. FOLTON, de MM. J. DUCHESNE et C. CUDENNEC ainsi que de M. G. BONTRON, Mme G. MENEZ et MM. D. DUBAND et JN. GAUTIER traitent chacune à leur manière de la transformation de la pluie en débit pour prévoir les débits d'étiage dans un réseau hydrographique ou pour évaluer les débits de crues et les risques d'inondation. Dans la quatrième communication, la démarche présentée par Mme P. BABILLOT et M. J. MARGAT est complètement différente et très originale. Elle consiste à faire de la comptabilité, c'est-à-dire à établir des bilans d'utilisation de l'eau en évaluant dans le temps et 
dans l'espace, en termes de flux et de variation de stocks, l'impact des utilisateurs. Cette démarche a pour objectif d'apporter aux aménageurs et aux gestionnaires les données leur permettant de traduire en termes économiques les influences des différents acteurs de l'eau pour faciliter les décisions d'investissement ou de gestion.

L'objectif de l'étude et des logiciels LOIEAU et SHYPRE présentés dans le rapport de MM. J. LAVABRE, P. ARNAUD et N. FOLTON, est de mettre à disposition des décideurs, une méthode d'estimation régionale de la ressource en eau dans un territoire couvrant les régions Provence-Alpes-Côte d'Azur, Languedoc-Roussillon et Corse soit $50000 \mathrm{~km}^{2}$.

L'analyse a porté sur 276 bassins versants et les données de 600 stations pluviométriques ont servi à caler les modèles.

Le point fort de cette communication est de confirmer l'intérêt d'utiliser l'information pluviométrique spatio-temporelle, plus abondante, pour simuler des chroniques de débit pour un bassin versant en s'appuyant sur des modèles simples et robustes, ce qui permet d'enrichir l'information hydrométrique partielle disponible.

A noter cependant qu'on ne supprime pas pour autant les aléas d'échantillonnage ni les problèmes de conditionnement des données dans le modèle.

Deux logiciels ont été élaborés sur ces bases.

Le logiciel LOIEAU incorpore des bases de données cartographiques, climatologiques et hydrologiques et génère en tout point du réseau hydrographique des chroniques de débits mensuels. Il permet une interpolation régionale pour des bassins non instrumentés en mesure de débit, et ainsi de reconstituer ou d'estimer des paramètres caractéristiques d'écoulement : module annuel, débit d'étiage quinquennal...

Cet outil très convivial permet aux utilisateurs d'apprécier les variations saisonnières et inter-annuelles de la ressource en eau dans le cadre de l'élaboration d'un SAGE. Son usage peut s'étendre également à l'étude de la faisabilité d'un réservoir d'eau ou à l'optimisation de la gestion de retenues existantes en le couplant avec le logiciel (SHYPRE) développé par cette équipe qui permet de prédéterminer des débits de crues par simulation de scénarios d'hydrogrammes.

Dans ce rapport, les auteurs ont mis en évidence qu'en utilisant à bon escient les informations hydrologiques et pluviométriques existantes et des modèles numériques courants et éprouvés et en les associant à des bases de données géographiques, il était possible de construire des outils originaux, robustes et conviviaux permettant de mieux aménager et mieux gérer la ressource en eau.

Bien que traitant du même sujet, la modélisation de la transformation pluie-débit, MM. J. DUCHESNE et C. CUDENNEC se sont attelés à un tout autre défi, celui de développer une fonction de transfert pluie-débit déterministe et polyvalente. La théorie développée par les auteurs s'appuie sur un raisonnement de physique statistique appliqué à un réseau hydrologique vu comme un objet fractal. Moyennant certaines précautions comme la vérification que le réseau respecte les propriétés d'homogénéité de géologie et de relief, ce modèle est mis en œuvre sans recours à aucun calage de paramètres.

Le modèle $\mathrm{H} 2 \mathrm{U}$ utilise une fonction de transfert brevetée qui transforme la pluie nette en un hydrogramme à l'exutoire d'un bassin versant, couplée à une fonction de production qui transforme la pluie brute en pluie nette par estimation des pertes. Cet hydrogramme est considéré comme unique et invariant et les auteurs utilisent une forme analytique dite " densité analytique des isochrones d'écoulement fluvial ", comparable à la fonction Gamma incomplète de NASH pour représenter cet hydrogramme mais avec une contrainte d'homogénéité du bassin et donc de limitation de la taille. II faut noter que le problème de la décomposition d'une fonction de transfert moyenne en fonctions de transfert propres à chaque sous-bassin homogène est un problème capital à résoudre pour tenir compte de certaines répartitions spatiales d'averses, car ces sous-bassins ne sont pas jaugés dans la plupart des cas.

Les auteurs citent des applications de cette méthode de modélisation mais sans en donner les résultats.

La méthode du gradex de calcul de la probabilité des crues rares et extrêmes est connue du monde entier par les publications et conférences dont elle a fait l'objet. G. BONTRON, G. MENEZ, D. DUBAND et JN. GAUTIER présentent dans leur rapport une application inhabituelle et très intéressante de cette méthode dans le cadre des études relatives à la sécurité des populations face au risque d'inondation du Plan Loire Grandeur Nature.

Les auteurs ont effectué un travail très fouillé d'analyse de la structure spatiale de la pluviométrie sur l'ensemble des bassins versants de la Loire et de l'Allier ainsi que des données historiques sur la forme des crues. Ils ont mis en évidence l'existence d'un hydrogramme représentatif des crues fortes à exceptionnelles. L'homogénéité des bassins versants a permis d'utiliser la méthode globalement sur l'ensemble du bassin de la Loire.

L'application de la méthode du gradex au bassin versant de la Loire $\left(32610 \mathrm{~km}^{2}\right)$ a permis de consolider la validité de la méthode dans le cas de grands bassins. Cette extension du domaine d'usage a été également utilisée avec succès à l'occasion de projets ou d'études hydrauliques à l'international.

En complément des références données par les auteurs, on peut citer la brochure sur le Gradex éditée par le CFGB en 1997.

Le rapport de P. BABILLOT et J. MARGAT expose la démarche qui a conclu à instaurer en France une comptabilité de l'eau et fait le point sur les applications en cours et les améliorations escomptées.

Après élaboration d'une méthode (présentée en 1986 dans les " comptes des eaux continentales " de l'INSEE) et un premier exercice dans un département, les comptes annuels ont été établis pour la France entière, pour une année de référence (1981). Ils sont présentés sous forme de tableaux de comptes de ressources et comptes d'utilisations et permettent de dégager des indicateurs en pourcentage.

Pour arriver à ces résultats en quantités d'eau, il a fallu unifier les références, subdiviser les systèmes hydrographiques et classer les agents utilisateurs. On peut aussi, en principe, assurer la liaison entre les comptes physiques et les comptes économiques.

Cette comptabilité analytique de l'eau est un instrument d'analyse intéressant : elle permet d'identifier les échanges et d'expliquer les variations de stocks et, grâce aux indicateurs appropriés, d'analyser et comparer différentes situations ; elle permet aussi de comparer dans des études prospectives les différentes utilisations envisagées. Il faut cependant se méfier de la validité et des incertitudes qui affectent certains chiffres comptables (prélèvements agri- 
coles, réserves souterraines, nivales, évaporation etc.).

Les auteurs indiquent fort judicieusement dans leur conclusion que l'approche comptable ne doit pas dispenser de l'analyse structurelle classique des ressources et des utilisations.

\subsection{Prévision et gestion des situations extrêmes}

Sept communications peuvent être classées dans ce thème. L'une d'entre elles, celle de JA. CUNGE, M. ERLICH et JL. RAHUEL traite de ce sujet parmi d'autres et sera analysée dans la dernière partie de l'exposé relatif à la gestion opérationnelle de l'eau.

La majorité des rapports fait état d'applications à l'international de méthodes et d'outils d'aide à la gestion des situations de crise provoquées par les excès climatiques démontrant ainsi leur caractère universel et l'influence de l'Ecole Française de l'Eau dans le monde.

Cinq d'entre eux ont en commun de présenter des développements d'outils informatiques d'assistance intégrant des systèmes d'acquisition de données ou d'information. Leur objectif est d'améliorer la qualité de la prévision et de permettre aux organisations responsables de la sécurité publique d'anticiper les actions préventives et de réagir rapidement en temps réel pour éviter des catastrophes.

Un rapport, celui de T. BOISSEAU et NG. CAMPHUIS, permet dans ses applications actuelles, d'évaluer l'efficacité des dispositifs d'écrêtement des crues constitués le long du lit d'un cours d'eau et de définir une stratégie de réduction des risques d'inondation.

Enfin, une des communications a trait à l'utilisation du système informatique TELEMAC pour la prévision des inondations dans les systèmes fluviaux complexes en plaine.

La prévision et l'annonce des crues est un exercice difficile surtout dans des bassins hydrographiques comme celui de la Garonne comme le montre le rapport de JJ. VIDAL, JP. DUPOUYET et T. MURILLO.

Ce rapport présente le système SOPHIE de suivi et de prévision des cotes et débits mis en place sur la Garonne et ses principaux affluents, par les services de l'Etat et une réflexion intéressante sur le choix de ses composants.

Le véritable point fort du système réside dans la structure informatique de collecte de données (cotes, débits, pluies) en temps réel et dans les logiciels de visualisation et traitement de cette information, à la fois modulaires et conviviaux. Son principal point faible semble être l'absence de moyens associés affichant l'information météorologique observée et prévue (météotel, sorties de modèles météo...).

Trois autres choix de composants du système SOPHIE méritent malgré tout réflexion, en effet :

- la procédure de décision multimodèle, bien que séduisante, avec " pondérations variables en fonction de l'état du système de prévision et des performances récentes des modèles ", apparaît peu réaliste surtout pour des crues rapides déjà difficiles à gérer avec un seul modèle,

- l'imagerie radar, très utile pour la localisation des averses, reste un sujet de recherche controversé quant à son utilisation pour le calcul des lames d'eau en zone montagneuse,

- le système multi-expert pour la prévision des crues en est encore au stade de la recherche avancée.

Il faut cependant encourager les services de l'Etat dans la mise en place d'un tel système en attirant leur attention sur le choix de concepts novateurs qui risquent de retarder le fonctionnement du système et par conséquent la diffusion le cas échéant de notre savoir-faire à l'international.

La communication de L. MOULIN et R. THEPOT présente la modernisation du système CRISTAL de collecte en temps réel de mesures de cotes de rivières et de pluies ponctuelles, opérationnel depuis 1985 dans le bassin de la Loire pour l'annonce des crues, le suivi des étiages et la gestion des réservoirs (VILLEREST, NAUSSAC...). Un retour d'expérience extrêmement fructueux a permis de préparer la refonte du système actuel avec des perspectives plus ambitieuses dans le cadre du Plan Loire Grandeur Nature.

L'objectif est de renouveler les équipements et d'améliorer la fiabilité du système, l'anticipation des prévisions et l'information des usagers, tout en maîtrisant les coûts d'investissement et de fonctionnement. L'architecture du nouveau système de collecte de données apparaît très pertinente, en étant répartie en centres locaux indépendants et un centre principal à Orléans. Par ailleurs, la séparation en 3 lots (stations de terrain, unités de collecte, unités de traitement) dans les cahiers des charges est tout à fait judicieuse.

La maintenance des systèmes de mesure, acquisition et transmission paraît avoir fait l'objet d'une étude approfondie. Le souci de développer un système d'aide à la décision de techniciens et ingénieurs en gestion mais également d'information pour le public des administrations et les élus est original. Il est sagement prévu d'étaler les mises en services de prototypes puis de systèmes locaux en testant leur fiabilité sur quelques années. D'autres développements concernant la prévision de crues d'étiage, la gestion multiusage des ressources en eau s'appuyant sur des systèmes d'aide sont également envisagés.

Tout ou partie de ces réalisations devraient servir à valoriser l'Ecole française de l'eau dans le monde. Les formations et compétences nécessaires des techniciens et ingénieurs devront cependant être à la hauteur des enjeux.

Les perspectives présentées dans la contribution de $\mathrm{T}$. BOISSEAU et N.G. CAMPHUIS sont originales et susceptibles de servir de référence.

En effet, il ne s'agit pas seulement d'élaborer un modèle hydraulique à grande échelle (plus de $1000 \mathrm{~km}$ de cours d'eau) permettant de tester pour différents scénarios de crues les faiblesses de l'endiguement actuel et les améliorations à y apporter, mais aussi d'inventorier les enjeux socio-économiques et de proposer des solutions pour réduire la vulnérabilité des zones exposées aux crues. Cette réflexion doit permettre d'engager une large concertation auprès des élus et de la population, pour prendre une décision et engager une solidarité amont-aval en Loire moyenne.

La Loire moyenne se prête bien à cette démarche car elle est aménagée depuis longtemps par un endiguement qui protège une partie importante du val des inondations occasionnées par des crues inférieures à la crue cinquantennale. Mais au-delà il peut $\mathrm{y}$ avoir franchissement des digues par des déverses ou des brèches plus ou moins aléatoires (renards) malgré l'existence de déversoirs de sécurité.

Le modèle hydraulique finement calé, composé d'un modèle filaire pour le réseau hydrographique et d'un modèle à casiers pour les vals et quartiers inondables permet par simulation d'améliorer la connaissance de la propagation des crues et ainsi, de diminuer la vulnérabilité de l'activité humaine et des biens.

La communication de A. GARCON, C. CARRE et P. 
LYAUDET aborde la problématique de la gestion des ressources en eau en cas de pénurie lors d'étiages et, lorsque l'on dispose de réserves d'eau par l'intermédiaire de barrages d'accumulation.

Cette gestion est particulièrement délicate face à la demande des usagers des secteurs énergie, irrigation, eau potable, piscicole, tourisme.

Dans le cadre des besoins spécifiques à EDF, deux exemples intéressants sont présentés développant l'aspect prévision des débits dans un processus de décision d'exploitation concernant la centrale nucléaire de Civaux sur la Vienne et celles de la Loire moyenne.

Pour la Vienne, la méthode de prévision à quelques jours s'appuie sur le modèle physique MORDOR (à 4 réservoirs et pas de temps journalier) de simulation des écoulements d'après les pluies pour différentes parties du bassin. Ce modèle est complété par un " recalage opérationnel " adaptation statistique tenant compte d'informations sur l'état présent, le proche passé et la prévision de pluie de Météo France. Ce système est complété par un outil d'aide à la décision de gestion SOVAGE.

Pour la Loire, la prévision est à plus long terme (deux à trois mois). Il s'agit d'une approche statistique s'appuyant sur les observations historiques de 110 années, et qui consiste à prévoir une courbe enveloppe de tarissement en fonction des conditions hydroclimatiques des mois précédant la période d'étiage (juin-septembre) et en améliorant cette distribution conditionnelle de la chronique des débits, par une sélection des années historiques analogues en hydraulicité de printemps.

Ces deux exemples montrent toute la difficulté de la prévision des faibles débits en un point donné d'un cours d'eau aménagé. Les incertitudes de cette prévision ont diverses origines : méconnaissance des prélèvements agricoles réels, rendement des lâchures de réservoirs en fonction de l'état des nappes phréatiques et aussi choix des modèles de simulation.

La communication de J.M. HERVOUET, M.D STEWART et P.D. BATES et Catherine WILSON, intitulée " Calibration and validation of two dimensional finite element models of floodplain flows " fait le point des réflexions et des études en cours à l'Université de BRISTOL (Angleterre) pour maîtriser les limitations inhérentes aux procédures de calibration et de validation utilisées dans la modélisation des écoulements des systèmes fluviaux complexes en plaine.

Une meilleure prévision des débits et des niveaux atteints dans de tels systèmes est l'objectif principal de cette recherche répondant à une attente certaine des ingénieurs de l'aménagement du territoire.

Les difficultés sont dues principalement à la complexité du modèle et au manque de données de validation. Aussi pour les surmonter, les auteurs ont-ils en particulier comparé les résultats d'un modèle élaboré à partir du système Telemac 2D avec un certain nombre de modèles physiques et avec des mesures de terrain effectuées sur la rivière Blackwater.

Les résultats de ces tests de comparaison ont mis en évidence en particulier les insuffisances des pratiques actuelles et le besoin d'améliorer la modélisation du processus d'inondation et d'assèchement des berges.

La communication d'A. VILLEVIELLE sur les catastrophes météo-hydrologiques de la zone Inde-Bangladesh nous fait prendre conscience de l'ampleur de la tâche qui reste à accomplir :

- par les chercheurs pour trouver les systèmes les mieux adaptés pour prévoir les crues, alerter les populations et réduire ainsi les risques liés aux inondations,

- par les Etats concernés et la collectivité internationale pour financer et rendre opérationnels ces systèmes.

Car tout est démesuré dans cette zone géographique située au pied du Toit du Monde et qui concerne l'Inde, le Népal, le Bangladesh et le Bhutan : l'intensité des pluies qui s'abattent sur ces régions, la misère extrême qui touche les populations, l'ampleur des inondations et des catastrophes qu'elles engendrent. Mais aussi, il faut le souligner, la mobilisation internationale qui s'est formée pour tenter de réduire les risques dus à l'eau et les pertes de vies humaines et de ressources alimentaires.

L'Union Internationale des Associations et Organismes Techniques (UATI) contribue à cette tâche à travers le projet SENTINEL qui comme son nom l'indique a pour objectif le développement d'un système informatique de prévision des crues faisant appel très largement aux satellites d'observation.

L'auteur met l'accent particulièrement sur la nécessité de mettre en commun les moyens de recherche internationaux pour faire aboutir ces projets.

\subsection{Connaissance et gestion des cours d'eau en dyna- mique}

Le thème de la dynamique des cours d'eau a suscité trois rapports et ces trois rapports se complètent remarquablement.

Les deux premiers s'intéressent à l'évolution morphologique des lits : celui de D. ROULT et M. RAMETTE, très didactique, rappelle les lois régissant les écoulements et les transports solides, avant d'aborder l'étude des évolutions morphologiques de grande ampleur ; celui de A. PAQUIER et P. RAMEZ souligne, dans un esprit très pragmatique, la nécessité de schématiser les données pour réussir une bonne représentation globale de l'évolution des lits. Les auteurs des deux rapports illustrent leurs propos par des exemples concrets.

Le troisième rapport présenté par $\mathrm{M}$. KASSOUL et $\mathrm{M}$. BELORGEY, concerne l'alluvionnement des barrages-réservoirs, aspect particulier de la dynamique des cours d'eau dont les conséquences environnementales et économiques sont considérables, et donne comme exemple un ensemble de barrages du Maghreb.

Le rapport de D. ROULT et M. RAMETTE comporte dans sa première partie un rappel très complet, et fort utile, sur la théorie des écoulements à surface libre et celle des transports solides. Si la première est bien connue des généralistes, de même que les lois du transport solide par charriage sur des fonds plats (applicables aux torrents et rivières à sédiments grossiers), celles relatives au cas général, c'est-àdire transport par charriage et suspension, avec formation de dunes, le sont beaucoup moins. Nous devons remercier les auteurs d'avoir dégagé parmi les nombreuses formules semiexpérimentales existantes, celles qui collent le mieux à la réalité des différents cas.

Lorsque les formules de ce genre se compliquent avec la multiplication des paramètres, que les grandeurs y figurent avec des exposants décimaux et que l'appréhension intuitive des réalités physiques devient difficile, les hydrauliciens ont depuis toujours le réflexe de recourir à des abaques. Dans ce 
domaine, l'emploi d'un abaque renseigne d'un coup d'œil sur la précision d'un résultat et permet des analyses de sensibilité souvent mieux que ne pourrait le faire un code de calcul.

L'abaque révèle aussi les zones où l'interprétation devient hasardeuse, lorsqu'on approche des limites de la représentation mathématique. Dans de tels cas, il y a lieu de se rabattre sur des approches empiriques. C'est pourquoi les auteurs rappellent fort opportunément les études anciennes de Lacey et la " théorie du régime", peu appliquée en France. Ils en font une analyse critique, montrant comment elles corroborent les approches plus théoriques, pourquoi elles sont particulièrement bien adaptées au cas des sédiments sableux et comment elles expliquent cette caractéristique remarquable des cours d'eau en équilibre morphologique : ils correspondent à une capacité maximale de transport solide par charriage, sur une pente minimale.

Dans sa deuxième partie, le rapport traite des cours d'eau " morphologiquement anormaux ", c'est-à-dire dont la pente est très forte, eu égard à la finesse de leurs sédiments. Ils citent l'exemple du Rio Piraï, en Bolivie, dont la pente sur $40 \mathrm{~km}$ en aval de Santa Cruz est 20 fois supérieure à la " normale ". Lors des crues, les affouillements sont considérables (10 mètres ou plus) puis, après l'épisode, les fonds reviennent à leur configuration initiale.

L'équation de Lacey traduit de manière satisfaisante le phénomène d'érosion et la profondeur d'affouillement.

Lorsque l'on a affaire à un cours d'eau comme celui-ci, la conception des ouvrages de protection contre les inondations est délicate dans les secteurs sensibles. Les ouvrages classiques sont exclus, il faut viser à guider le flot au moyen d'épis perméables constitués de pieux reliés par du grillage et, pour le reste, " faire la part de l'eau " c'est-à-dire localiser dans les secteurs moins sensibles les inévitables évolutions du lit.

Le rapport de A. PAQUIER et P. RAMEZ met l'accent sur 4 points-clés pour l'étude de l'évolution du lit d'un cours d'eau :

- la distinction entre rivière à sable et rivière à gravier : plus précisément, en fonction du paramètre rugosité relative $(\mathrm{D} / \mathrm{H})$, la classification suivante est proposée : rivières à dunes (rivières à sable et mixtes à comportement sableux) et rivières à comportement dynamique (rivières à gravier et mixtes à comportement graveleux),

- la prise en compte des degrés de liberté : la rivière compense un déficit ou un excès de charge solide en ajustant la morphologie de ses tronçons,

- la description synthétique de la topographie, par découpage en tronçons homogènes articulés par des discontinuités, - la simplification des processus, en vue de la modélisation de l'écoulement liquide et de la déformation résultant du transport solide.

L'application citée est l'étude de l'Arc en Maurienne caractérisé par une forte pente et de nombreuses discontinuités. Elle a comporté l'estimation du diamètre caractéristique des grains de la couche active du substrat, la délimitation des tronçons homogènes, puis le lissage de la géométrie et le filtrage des contraintes. Après calage du modèle, on peut prédire l'évolution du lit sur les $3 \mathrm{~km}$ du tronçon analysé, pour différentes crues.

Le rapport de M. KASSOUL et M. BELORGEY examine la gestion de l'alluvionnement sur un échantillon de 19 barrages algériens. L'ensemble de ces réservoirs (mis en service entre 1932 et 1978) avait perdu en $1986,44 \%$ de sa capacité initiale.

Le rapport présente les caractéristiques géométriques et hydromorphiques des réservoirs et des bassins versants. Il indique comment ont été réalisées les mesures bathymétriques (échosondeur enregistrant en continu).

La mise en forme des données a privilégié le taux d'abrasion des bassins versants $\left(\mathrm{m}^{3} / \mathrm{km}^{2} / \mathrm{an}\right)$, le coefficient d'écoulement et le coefficient d'altitude et, en fonction de ces paramètres, les auteurs ont classé les barrages en 3 catégories.

Après cette analyse de l'envasement des barrages anciens, ils ont cherché à évaluer la durée de vie de 11 barrages récemment mis en service (de 1984 à 1988). Pour 3 d'entre eux, dont la durée de vie est courte (20 à 100 ans), ils ont recommandé d'élaborer un protocole d'exploitation particulier.

Enfin, les auteurs ont comparé l'échantillon des 19 barrages algériens à 7 barrages tunisiens et 16 barrages marocains sujets à des envasements comparables et l'une de leurs recommandations est l'élaboration d'un fichier maghrébin d'exploitation des retenues en vue d'actualiser les bilans hydrauliques.

Les auteurs donnent une liste bibliographique qui pourrait être complétée utilement par de nombreuses autres références récentes. Plusieurs associations techniques internationales se préoccupent de l'alluvionnement dans les retenues et, parmi leurs publications, on peut citer :

- le Bulletin $n^{\circ} 67$ (1989) de la Commission Internationale des Grands barrages : " Maîtrise de l'alluvionnement des retenues. Recommandation" ainsi que les travaux en cours du Comité technique de l'alluvionnement de la CIGB,

- les travaux de l'ICCORES, groupe de coordination de plusieurs associations internationales (CIGB, AIRH, CIID, etc.) et notamment les actes de la dernière Conférence Internationale sur Alluvionnement (Fort Collins, sept. 1996) et de la réunion d'août 1997.

\subsection{Connaissance et gestion des écosystèmes aqua- tiques}

Cinq rapports sont présentés sur ce thème. Leur richesse et leur diversité suffisent à montrer l'importance qui est accordée aux questions touchant à l'environnement.

Les deux premiers rapports sont relatifs à des aménagements en service et montrent comment ont été traités un projet de réhabilitation (le vieux Rhône à l'aval de Pierre Bénite) et une difficulté à la mise en eau nécessitant de réoxygéner l'eau à l'aval d'un barrage (Petit Saut en Guyane).

Les trois autres sont plus généraux et axés sur la modélisation des écoulements et de phénomènes physiques ou biologiques complexes : réhabilitation de tronçons de la Loire, réhabilitation de sites pollués et recherches diverses en hydroécologie.

Le rapport de P. MICHELET, C. TERRIER et M. VOLLE décrit le projet de réhabilitation écologique du Vieux Rhône de Pierre Bénite. La mise en service de l'aménagement de la Compagnie Nationale du Rhône avait profondément affecté le paysage, la faune et la flore à l'aval du barrage. Le site possédait cependant une forte identité paysagère, une faune assez remarquable et un potentiel de diversification intéressant.

L'étude de la réhabilitation a été engagée en 1991, avec comme objectif le retour à un fonctionnement local proche 
de celui qui existait avant aménagement. Le projet comporte :

- l'augmentation du débit dans le Rhône court-circuité : le débit moyen naturel étant de $1000 \mathrm{~m}^{3} / \mathrm{s}$, le débit réservé actuel est de 10 ou $20 \mathrm{~m}^{3} / \mathrm{s}$; l'étude a montré que $100 \mathrm{~m}^{3 / 5}$ constitue le minimum pour créer des conditions naturelles propres à revitaliser ce tronçon. L'augmentation du débit est réalisable sans perte de production hydroélectrique grâce à l'installation d'une petite centrale hydraulique et sans modifications des conditions de la navigation.

- la restauration de trois lônes avec recreusement ou recalibrage, permettant de créer un ensemble de milieux humides complémentaires.

- la mise en valeur des espaces riverains à destination du public.

Un suivi scientifique portant sur la qualité des eaux, la flore et la faune sera assuré par une équipe multidisciplinaire.

Le financement du projet, estimé à $100 \mathrm{MF}$, est assuré par la CNR $(40 \%)$, l'Agence de l'Eau $(40 \%)$ et le Syndicat Mixte Intercommunal du Rhône (20\%).

Ce projet ambitieux et novateur devrait être en service fin 1999. L'expérience pourrait être reconduite sur 4 à 8 sites du Rhône.

Les dispositions exceptionnelles mises en æuvre lors du remplissage puis du début d'exploitation de l'aménagement de Petit Saut, sous le contrôle d'un Comité Scientifique indépendant, ont déjà fait l'objet de nombreuses publications et conférences. Le présent rapport, qui intègre les résultats des campagnes 1997 et 1998, constitue une synthèse des observations faites depuis janvier 1994 et permet de porter un jugement sur l'efficacité des solutions mises en œuvre pour améliorer la qualité de l'eau à l'aval de l'aménagement.

Le premier constat a été celui d'une anoxie rapide dans le lac dès la mise en eau et d'une désoxygènation à l'aval plus importante que prévu. Par deux fois en 1994, le manque d'oxygène dissous a provoqué la mort de poissons.

Un dispositif de réoxygénation artificielle a été mis en service en février 1995. Il consiste en un seuil déversant à deux chutes doublé d'un système d'oxygénation forcée. Ce dernier s'est révélé inefficace mais le seuil a donné de très bons résultats quant à l'élimination du méthane et la réoxygénation.

A partir des données recueillies sur la composition physico-chimique des différentes veines d'eau transitant par les turbines, le déversoir et les évacuateurs de fond, des consignes d'exploitation ont été élaborées et adaptées à l'amélioration progressive (épaississement de la couche épiliminique) de la retenue.

L'exemple de Petit Saut montre que, s'il est difficile d'agir sur les paramètres d'une retenue de ce type, une gestion adéquate du barrage permet de bien réguler la qualité physico-chimique à l'aval.

Le rapport de G. ROUAS et JM. TANGUY rappelle que, pour remédier aux détériorations consécutives aux extractions d'alluvions et aux aménagements de navigation, le plan " Loire grandeur nature " consiste en une restauration du lit visant à améliorer la capacité d'écoulement des crues, relever la ligne d'eau d'étiage pour remettre en eau les bras secondaires et réhabiliter les milieux naturels.

La Loire moyenne (400 km entre le Bec d'Allier et le Bec de Maine) présente généralement une morphologie de bras multiples séparés par des îles stabilisées et des bancs instables.

Les études de restauration du lit ont débuté sur un tronçon prioritaire, celui du secteur de la Charité-sur-Loire. Elles reposent sur une modélisation $2 \mathrm{D}$ réalisée à l'aide du logiciel hydrodynamique REFLUX. Le rapport détaille les précautions prises pour la collecte des données bathymétriques, topographiques et hydrologiques, pour la construction du modèle (partition en zones et affectation des rugosités) et pour son calage (sur 2 crues de fréquence biennale et un épisode de basses eaux).

Le modèle a été exploité ensuite pour des crues de 10 à 100 ans, en testant différentes solutions d'aménagement " douces " (enlèvement d'atterrissements) ou plus "lourdes" (creusement d'un chenal dans le bras secondaire et rabotage d'une île). Comme on pouvait s'y attendre, seules ces dernières sont de nature à apporter une amélioration notable.

En conclusion, les auteurs tentent d'établir les domaines de validité des modèles mono et bidimensionnels pour ce type de problème. Ils préconisent l'emploi du modèle 2D sur des tronçons d'une vingtaine de kilomètres, lorsqu'on veut balayer toute l'échelle des débits, ce dont on peut aisément convenir.

Le rapport de J.-M. COME et P. Le THIEZ est consacré au logiciel SIMUSCOPP, code tridimentionnel simulant la migration de polluants dans un milieu souterrain poreux. II résout les équations d'écoulement en triphasique pour un mélange à plusieurs constituants et prend en compte différents modes de transfert et de dégradation.

La validation du code a été effectuée en simulant la migration d'un volume de gazole dans un bassin parallélépipédique de grandes dimensions, rempli de sable, avec un niveau phréatique réglable, l'extension de la zone polluée étant contrôlée par des prélèvements de sol.

Le rapport cite deux applications à des cas réels de pollution accidentelle, visant à prévoir leurs conséquences et déterminer les remèdes :

- pollution de nappe phréatique consécutive à une fuite de fuel sur un oléoduc : après une migration verticale rapide, le polluant s'est étalé sur la nappe. La riposte appropriée a été un rabattement et un écrémage de la phase et un confinement à partir de tranchées de réinfiltration. Le modèle a permis d'optimiser le dispositif hydraulique de récupération, décider l'arrêt des pompages au bon moment et prévoir les concentrations en hydrocarbure à l'aval du site.

- réhabilitation d'un aquifère fortement pollué par des hydrocarbures et des solvants chlorés : la simulation a conduit à préconiser un dispositif de dépollution par forages et a quantifié le débit de pompage, la localisation des forages et la durée à prévoir pour le traitement (a priori, plusieurs décennies).

Ces deux exemples montrent à l'évidence les services que peut rendre l'outil SIMUSCOPP.

Le rapport de P. GOSSE retrace I'historique de la recherche en hydroécologie à EDF, depuis le début des années 1960. Initiée avec l'étude de l'impact du projet de la centrale thermique de Montereau sur les peuplements piscicoles, cette recherche connut une activité intense lors de la préparation du programme électronucléaire et se diversifia dans les différents domaines de l'hydroécologie.

L'équipe Eau du Département Environnement, forte d'une vingtaine de personnes depuis 1980, étudie l'impact de la 
gestion des ouvrages sur les écosystèmes aquatiques (échauffement artificiel, transfert des micropolluants et radioéléments, problèmes spécifiques des barrages et des retenues et inversement l'impact du milieu sur le fonctionnement. des ouvrages (développement d'organismes aquatiques aux prises d'eau, par exemple).

En ce qui concerne le régime thermique des cours d'eau, furent mis au point successivement un modèle de calcul de la température d'une rivière de plaine, puis les premiers modèles non permanents sur de longs tronçons, avec application au Rhône et au Rhin. Ensuite furent développés des modèles adaptés aux cas de la Gironde, de la Manche et des barrages-réservoirs.

Un gros effort fut consacré à la recherche sur l'eutrophisation à l'occasion des études d'impact de Porcheville, des études sur le développement aval du Lot et du Doubs, puis sur la Durance et le Verdon. Un code de calcul standard de l'évolution de la biomasse phytoplanctonique fut mis au point dans les années 1980. Cet effort fut étendu aux milieux lacustres pour les besoins de l'exploitation des retenues (Grangent, Pareloup,...) et aux milieux marins (ensemble de la Manche et conséquences de l'apport d'azote dans le Golfe normand-breton).

Une autre question sensible, le comportement des poissons migrateurs, a également mobilisé les chercheurs, qui ont développé une méthode d'évaluation de l'impact du débit sur les peuplements piscicoles en stade non migratoire et un programme approfondi sur la conception des passes à poissons.

Dans le domaine de la contamination des rivières par des éléments traces, la recherche a porté sur les cinétiques d'accumulation/relargage des métaux et radionucleïdes et le transfert de radioactivité via les écosystèmes.

Comme dernier exemple, dans le cadre de la gestion intégrée de la ressource en eau, on cite le logiciel AGIRE qui permet de déterminer à l'échelle d'un bassin versant l'impact des scénarios de gestion pour la satisfaction à long terme des différents usages de l'eau.

L'auteur souligne dans sa conclusion la difficulté particulière inhérente à la composante biologique : elle oblige à dégager les mécanismes dominants et elle fait obstacle à la définition de méthodes standards. Il faut une grande expérience pour choisir le juste niveau d'investigation dans les multiples disciplines concernées et pour être en mesure d'apporter des réponses pertinentes dans des délais raisonnables.

Dans les domaines complexes de l'hydroécologie, le succès est dû pour beaucoup à la continuité de l'effort et à la coopération étroite entre les concepteurs et les exploitants des centrales.

Cette rétrospective sur les vingt-cinq ans de recherche en hydroécologie appliquée intéressante et cohérente, est tout à fait dans l'esprit de ces 25 èmes Journées.

\section{- 3.5. Gestion opérationnelle de l'eau}

Les deux rapports présentés dans ce thème couvrent des aspects distincts : l'intégration des systèmes informatiques, les technologies télématiques avancées et la gestion de réseaux isolés de production hydroélectrique.

Le rapport de P. ROUSSET et F. BONNADIER nous décrit l'évolution de la Société du Canal de Provence au long de 3 décennies pendant lesquelles ses systèmes informatiques de gestion, après s'être développés séparément, se sont progressivement intégrés.
Comme ce fut souvent le cas, l'activité de facturation fut informatisée en premier. A partir de 1970 fut mise en place la régulation automatique centralisée des ouvrages principaux, puis de tous les ouvrages de stockage, transport, traitement des eaux, et distribution, grâce à un système dynamique intégrant la prévision de la demande et l'optimisation de la production.

Au cours des années 1980, ce fut le tour des activités de maintenance, avec la gestion des inventaires, l'ordonnancement des interventions préventives et le suivi des coûts. Parallèlement, les tâches de conception, de suivi des projets et de gestion technique ont fait appel de plus en plus à la DAO et à la CAO.

A ce stade, la nécessité d'organiser les échanges d'information entre les différentes activités, pour mieux garantir la cohérence et améliorer les performances d'ensemble est apparue comme une évidence. Une étape décisive fut franchie à l'occasion du projet européen WATER CIME, auquel la SCP participa comme entreprise pilote. Le consortium WATER CIME a développé une méthodologie visant à apporter des réponses opérationnelles au problème de l'intégration pour les besoins des sociétés de distribution d'eau. Ses travaux se sont concrétisés par une plate-forme expérimentale qui démontre que l'intégration des outils de gestion classiques est réalisable et qu'elle permet une amélioration des performances de ces outils.

Dans sa réflexion, la SCP a suivi en permanence une démarche prudente et pragmatique : elle a exclu les solutions de refonte complète des systèmes et s'est engagée dans un processus d'intégration progressive.

Le rapport de J.A. CUNGE, M. ERLICH et J.L. RAHUEL présente l'éventail des activités du LHF qui ont donné lieu à des actions de Recherche et Développement et à des transferts de technologie dans le domaine de la télématique pour l'environnement. Sont cités à ce titre :

- Un programme-cadre de l'Union Européenne (4ème PCRD du secteur " Télématique et Environnement "), en cours de réalisation. Parmi les 26 projets retenus sur appel d'offres, 12 traitent de la ressource en eau.

- Deux systèmes hydroinformatiques opérationnels, l'un mis en œuvre pour la prévision du risque d'inondation à Shanghaï, l'autre relatif aux crues éclairs en France, en cours d'essai sur deux sites-tests et dont les premiers résultats sont jugés prometteurs.

- Un système d'information avancé portant sur la pollution agricole, avec en question de fond : " comment concilier l'utilisation des engrais et pesticides et les risques de contamination des ressources en eau ?". Cette question ouvre le débat sur la validité des études dans le domaine de la ressource en eau et il semble que la réflexion technique cède le pas à la réflexion sur les moyens de faciliter le dialogue entre les acteurs défendant des intérêts contradictoires.

- L’emploi généralisé du modèle d'écoulement bidimensionnel TELEMAC 2D, bien adapté aux géométries complexes et désormais diffusé largement à des licenciés français ou étrangers.

Dans leur conclusion, les auteurs soulignent l'intérêt commercial de procéder à des transferts de technologies de pointe vers les pays en développement et nous pourrions ajouter de porter haut le prestige de I'Ecole Française de l'Eau dans cette recherche mondiale d'un développement durable. 